\title{
Limit cycles bifurcating from a 2-dimensional isochronous torus in $\mathbb{R}^{3}$
}

\author{
Jaume Llibre*, Salomón Rebollo-Perdomo and \\ Joan Torregrosa* \\ Departament de Matemàtiques, \\ Universitat Autònoma de Barcelona, \\ 08193 Bellaterra (Barcelona) \\ Spain \\ e-mail: jllibre@mat.uab.cat,srebollo@mat.uab.cat,torre@mat.uab.cat
}

June 2, 2010

\begin{abstract}
In this paper we illustrate the explicit implementation of a method for computing limit cycles which bifurcate from a 2-dimensional isochronous set contained in $\mathbb{R}^{3}$, when we perturb it inside a class of differential systems. This method is based in the averaging theory. As far as we know all applications of this method have been made perturbing noncompact surfaces, as for instance a plane or a cylinder in $\mathbb{R}^{3}$. Here we consider polynomial perturbations of degree $d$ of an isochronous torus. We prove that, up to first order in the perturbation, at most $2(d+1)$ limit cycles can bifurcate from a such torus and that there exist polynomial perturbations of degree $d$ of the torus such that exactly $\nu$ limit cycles bifurcate from such a torus for every $\nu \in\{2,4, \ldots, 2(d+1)\}$.
\end{abstract}

2000 Mathematics Subject Classification. 34C29, 37G15, 34C25.

Key words. Limit cycle, periodic orbit, isochronous center, averaging method.

\section{Introduction}

Let $\mathbb{T}$ be a 2-dimensional torus of an integrable Hamiltonian system of two degrees of freedom contained in a 3-dimensional energy level and foliated by periodic orbits.

*Partially supported by the MICIIN/FEDER grant number MTM2008-03437 and by the Generalitat de Catalunya grant number 2009SGR410. 
Under the assumptions of the Poincaré-Birkhoff Theorem we can study the periodic orbits of $\mathbb{T}$ which persist when we perturb this integrable system inside the class of all Hamiltonian systems of two degrees of freedom, for details see [1, 2].

Assume now that $\mathbb{T}$ is a 2-dimensional isochronous torus of a differential system in $\mathbb{R}^{3}$, and that is $\mathbb{T}$ is foliated by periodic orbits with the same period. We also suppose that there are no other invariant tori in a neighborhood of $\mathbb{T}$. We want to study how many periodic orbits of $\mathbb{T}$ persist when we perturb it inside a class of differential systems of $\mathbb{R}^{3}$. Clearly we cannot apply the Poincaré-Birkhoff Theorem because it only works when we perturb energy levels of an integrable Hamiltonian system, and in such energy levels the invariant tori with periodic orbits are not isolated. In other words our problem is far removed from Hamiltonian dynamics.

In general we can consider a differential system in $\mathbb{R}^{3}$, with associated vector field $X_{0}$, having a 2-dimensional isochronous subset $\mathcal{S}$, that is $\mathcal{S}$ is comprised of periodic orbits with the same period. If we take the vector field $X_{\varepsilon}=X_{0}+\varepsilon X$ as an $\varepsilon$-perturbation of $X_{0}$, then natural questions are: Has the vector field $X_{\varepsilon}$ periodic orbits or limit cycles emerging from $\mathcal{S}$ ? How to compute them? How many? These questions can be considered analogous to the following about the theory of limit cycles of planar differential systems: How many limit cycles emerge under a perturbation from a center? Many authors have lately been attracted by this subject and succeeded in obtaining very interesting results (see for example the book [5] and the references therein).

A tool for studying these problems is averaging theory. For a general introduction to this theory see the books of Sanders and Verhulst [14], and of Verhulst [15], and for concrete applications see, for example, $[4,9,10]$ and $[6]$ for an application to a non- $\mathcal{C}^{1}$ differentiable system.

The perturbation of planes or cylinders inside $\mathbb{R}^{3}$ with a continuum of periodic orbits of a differential system has been studied using averaging theory in $[8,10]$ and using other techniques in [7]. As far as we know this is the first time that perturbations of an isolated compact surface foliated by periodic orbits with the same period have been analyzed.

We illustrate the method of studying the limit cycles that bifurcate from the isochronous periodic orbits comprising a 2-dimensional torus of a differential system in $\mathbb{R}^{3}$ with the following example. But the method can be applied to any differential system under similar assumptions.

In this paper we consider the differential system

$$
\begin{aligned}
& \dot{x}=\left(\left(\sqrt{x^{2}+y^{2}}-2\right) f(x, y, z)-z\right) \frac{x}{\sqrt{x^{2}+y^{2}}}+\varepsilon P(x, y, z), \\
& \dot{y}=\left(\left(\sqrt{x^{2}+y^{2}}-2\right) f(x, y, z)-z\right) \frac{y}{\sqrt{x^{2}+y^{2}}}+\varepsilon Q(x, y, z), \\
& \dot{z}=z f(x, y, z)+\left(\sqrt{x^{2}+y^{2}}-2\right)+\varepsilon R(x, y, z),
\end{aligned}
$$

defined in $\mathbb{R}^{3} \backslash\{(0,0, z) \mid z \in \mathbb{R}\}$, where $f(x, y, z)=1-\left(\sqrt{x^{2}+y^{2}}-2\right)^{2}-z^{2}, \varepsilon$ 
is a small parameter, and $P(x, y, z), Q(x, y, z)$ and $R(x, y, z)$ are polynomials. As usual the dot denotes differentiation with respect to the variable $t$.

The 2-dimensional torus

$$
\mathbb{T}:=\left\{(x, y, z) \in \mathbb{R}^{3} \mid f(x, y, z)=0\right\}
$$

is an invariant surface for the unperturbed system $\left(1_{0}\right)$. In addition, the solution of $\left(1_{0}\right)$ passing through the point

$$
\left(\left(2+\cos \theta_{0}\right) \cos \phi_{0},\left(2+\cos \theta_{0}\right) \sin \phi_{0}, \sin \theta_{0}\right) \in \mathbb{T}
$$

at time $t=0$ is

$$
x(t)=\left(2+\cos \left(t+\theta_{0}\right)\right) \cos \phi_{0}, \quad y(t)=\left(2+\cos \left(t+\theta_{0}\right)\right) \sin \phi_{0}, z(t)=\sin \left(t+\theta_{0}\right),
$$

so it is periodic with period $2 \pi$. This means that $\mathbb{T}$ is isochronous.

For $\varepsilon$ sufficiently small we study how many limit cycles of system $\left(1_{\varepsilon}\right)$ bifurcate from the periodic orbits of the invariant torus $\mathbb{T}$ of $\left(1_{0}\right)$; we recall that a periodic orbit of a differential system is a limit cycle if it is isolated in the set of all periodic orbits of the system. We do not provide a complete answer, we only study the bifurcated limit cycles which are controlled up to first order in $\varepsilon$ by using averaging theory.

The idea is as follows. We apply the ideas of averaging theory and an appropriate system of coordinates $(\theta, \phi, r)$ in an open neighborhood of $\mathbb{T}$ (see Lemma 4 ) to reduce system $\left(1_{\varepsilon}\right)$ to the form

$$
\mathbf{x}^{\prime}(\theta)=F_{0}(\theta, \mathbf{x})+\varepsilon F_{1}(\theta, \mathbf{x})+\varepsilon^{2} F_{2}(\theta, \mathbf{x}, \varepsilon),
$$

where the prime denotes the differentiation with respect to the variable $\theta$, and the functions $F_{0}, F_{1}$ and $F_{2}$ are $\mathcal{C}^{2}$ functions and $2 \pi$-periodic in the variable $\theta$. We will see in Section 3, by using techniques described in Section 2, that the number of limit cycles of system $\left(1_{\varepsilon}\right)$ bifurcating from the periodic orbits of the invariant torus $\mathbb{T}$ of $\left(1_{0}\right)$ are controlled up to first order in $\varepsilon$ by the number of simple zeros of a function $\mathcal{F}(\phi)$ defined in Theorem 2, which in our case is

$$
\mathcal{F}(\phi)=\int_{0}^{2 \pi} \frac{\widetilde{Q}(\theta, \phi, 1) \cos \phi-\widetilde{P}(\theta, \phi, 1) \sin \phi}{2+\cos \theta} d \theta
$$

where $\phi \in \mathbb{S}^{1}:=\mathbb{R} /(2 \pi \mathbb{Z})$, and $\widetilde{P}(\theta, \phi, 1)$ and $\widetilde{Q}(\theta, \phi, 1)$ are the expressions in the new coordinates of the polynomials $P(x, y, z)$ and $Q(x, y, z)$ of $\left(1_{\varepsilon}\right)$ restricted to the torus $\mathbb{T}$ of $\left(1_{0}\right)$ respectively. Hence as we shall see our problem reduces to studying how many simple zeros have this function $\mathcal{F}(\phi)$. The results of averaging theory guarantee the existence of a hyperbolic limit cycle for each simple zero of this function. We recall that a limit cycle $\gamma$ of a differential system is hyperbolic when all its (nontangent) characteristic exponents, that is the eigenvalues of the derivative of the return map associated to $\gamma$, have nonzero real parts.

If this function $\mathcal{F}(\phi)$ vanishes identically, then results using higher order averaging theory in $\varepsilon$ must be applied.

Our main result is the following. 
Theorem 1 For $\varepsilon \neq 0$ sufficiently small the following statements hold.

(i) If the maximum of the degrees of the polynomials $P(x, y, z)$ and $Q(x, y, z)$ is $d$ and $\mathcal{F}(\phi)$ given by $(2)$ does not vanish identically, then $2(d+1)$ is the maximum number of limit cycles of system $\left(1_{\varepsilon}\right)$ that can bifurcate from the periodic orbits of the torus $\mathbb{T}$ of system $\left(1_{0}\right)$.

(ii) For any natural $d$ and any $\nu$ in the set $\{2,4, \ldots, 2(d+1)\}$, we can find polynomials $P(x, y, z)$ and $Q(x, y, z)$ of degree $d$ such that system $\left(1_{\varepsilon}\right)$ has exactly $\nu$ limit cycles, which all are hyperbolic, bifurcating from the periodic orbits of the torus $\mathbb{T}$ of system $\left(1_{0}\right)$.

We note that this result does not depend on the polynomial $R(x, y, z)$. This is due to the fact that the function $\mathcal{F}(\phi)$ given by $(2)$ is independent of $R(x, y, z)$.

The paper is structured as follows. In Section 2 we summarize the result from averaging theory that we will use for proving Theorem 1. In Section 3 we prove Theorem 1.

\section{Averaging theory}

We consider the problem of the bifurcation of $T$-periodic orbits from the differential system

$$
\dot{\mathbf{x}}(t)=F_{0}(t, \mathbf{x})+\varepsilon F_{1}(t, \mathbf{x})+\varepsilon^{2} F_{2}(t, \mathbf{x}, \varepsilon),
$$

with $\varepsilon \in\left(-\varepsilon_{0}, \varepsilon_{0}\right)$ and $\varepsilon_{0}>0$ sufficiently small. The functions $F_{0}, F_{1}: \mathbb{R} \times \Omega \rightarrow \mathbb{R}^{n}$ and $F_{2}: \mathbb{R} \times \Omega \times\left(-\varepsilon_{0}, \varepsilon_{0}\right) \rightarrow \mathbb{R}^{n}$ are $\mathcal{C}^{k}$ functions, with $k \geq 2$ and $T$-periodic in the variable $t . \Omega$ is an open subset of $\mathbb{R}^{n}$. In addition, our main assumption is that the unperturbed system $\left(3_{0}\right)$ has a $m$-dimensional submanifold, with $1 \leq m \leq n$, comprised of $T$-periodic orbits.

Let $\mathbf{x}(t, \mathbf{z})$ be the solution of the unperturbed system $\left(3_{0}\right)$ such that $\mathbf{x}(0, \mathbf{z})=\mathbf{z}$. We write the linearization of $\left(3_{0}\right)$ along the periodic solution $\mathbf{x}(t, \mathbf{z})$ as

$$
\dot{y}(t)=D_{x} F_{0}(t, \mathbf{x}(t, \mathbf{z})) y .
$$

In what follows we denote by $M_{\mathbf{z}}(t)$ a fundamental matrix of the linear differential system (4), by $\xi: \mathbb{R}^{m} \times \mathbb{R}^{n-m} \rightarrow \mathbb{R}^{m}$ and $\xi^{\perp}: \mathbb{R}^{m} \times \mathbb{R}^{n-m} \rightarrow \mathbb{R}^{n-m}$ the projections of $\mathbb{R}^{n}$ onto its first $m$ and $n-m$ coordinates respectively; i.e. $\xi\left(x_{1}, \ldots, x_{n}\right)=$ $\left(x_{1}, \ldots, x_{m}\right)$, and $\xi^{\perp}\left(x_{1}, \ldots, x_{n}\right)=\left(x_{m+1}, \ldots, x_{n}\right)$

Theorem 2 Let $V \subset \mathbb{R}^{m}$ be open and bounded and let $\beta_{0}: \mathrm{Cl}(V) \rightarrow \mathbb{R}^{n-m}$ be a $\mathcal{C}^{k}$ function. We assume that

(a) $\mathcal{Z}=\left\{\mathbf{z}_{\alpha}:=\left(\alpha, \beta_{0}(\alpha)\right) \mid \alpha \in \mathrm{Cl}(V)\right\} \subset \Omega$ and that for each $\mathbf{z}_{\alpha} \in \mathcal{Z}$ the solution $\mathbf{x}\left(t, \mathbf{z}_{\alpha}\right)$ of $\left(3_{0}\right)$ is T-periodic; and that 
(b) for each $\mathbf{z}_{\alpha} \in \mathcal{Z}$ there is a fundamental matrix $M_{\mathbf{z}_{\alpha}}(t)$ of (4) such that the matrix $M_{\mathbf{z}_{\alpha}}^{-1}(0)-M_{\mathbf{z}_{\alpha}}^{-1}(T)$ has in the upper right corner the $m \times(n-m)$ zero matrix, and in the lower right corner $a(n-m) \times(n-m)$ matrix $\Delta_{\alpha}$ with $\operatorname{det}\left(\Delta_{\alpha}\right) \neq 0$.

Consider the function $\mathcal{F}: \mathrm{Cl}(V) \rightarrow \mathbb{R}^{m}$

$$
\mathcal{F}(\alpha)=\xi\left(\int_{0}^{T} M_{\mathbf{z}_{\alpha}}^{-1}(t) F_{1}\left(t, \mathbf{x}\left(t, \mathbf{z}_{\alpha}\right)\right) d t\right)
$$

Suppose that there is $\alpha_{0} \in V$ with $\mathcal{F}\left(\alpha_{0}\right)=0$, then the following statements hold.

(i) If $\operatorname{det}\left((\partial \mathcal{F} / \partial \alpha)\left(\alpha_{0}\right)\right) \neq 0$ then, for $\varepsilon$ small enough, there is a unique $T$-periodic solution $\varphi_{1}(t, \varepsilon)$ of system $\left(3_{\varepsilon}\right)$ such that $\varphi_{1}(t, \varepsilon) \rightarrow \mathbf{x}\left(t, \mathbf{z}_{\alpha_{0}}\right)$ as $\varepsilon \rightarrow 0$.

(ii) If $m=1$ and $\mathcal{F}^{\prime}\left(\alpha_{0}\right)=\cdots=\mathcal{F}^{(s-1)}\left(\alpha_{0}\right)=0$ and $\mathcal{F}^{(s)}\left(\alpha_{0}\right) \neq 0$, with $s \leq k$, then, for $\varepsilon$ small enough, there are at most $s T$-periodic solutions, $\varphi_{1}(t, \varepsilon), \ldots, \varphi_{s}(t, \varepsilon)$, of system $\left(3_{\varepsilon}\right)$ such that $\varphi_{i}(t, \varepsilon) \rightarrow \mathbf{x}\left(t, \mathbf{z}_{\alpha_{0}}\right)$ as $\varepsilon \rightarrow 0$ for $i=1, \ldots, s$.

The statement $(i)$ goes back to Malkin [12] and Roseau [13], for a shorter proof see [3]. The statement $(i i)$ is a refinement of $(i)$ for the case $m=1$, and since for proving it we need to recall ideas of the proof of $(i)$, we provide the complete proof of Theorem 2, and as a consequence of its proof the next result follows.

Corollary 3 Under the hypothesis of Theorem 2, if all the eigenvalues of the matrices $\Delta_{\alpha_{0}}$ and $(\partial \mathcal{F} / \partial \alpha)\left(\alpha_{0}\right)$ have nonzero real part, then the T-periodic solution $\varphi_{1}(t, \varepsilon)$ of Theorem 2. (i) is hyperbolic.

Proof of Theorem 2. From (a) there exist an open neighborhood $D$ of $\mathcal{Z}$ in $\Omega$ and $0<\varepsilon_{1} \leq \varepsilon_{0}$ such that any solution $\mathbf{x}(t, \mathbf{z}, \varepsilon)$ of $\left(3_{\varepsilon}\right)$ with initial conditions in $D \times\left(-\varepsilon_{1}, \varepsilon_{1}\right)$ is well defined in $[0, T]$. We consider the function $f: D \times\left(-\varepsilon_{1}, \varepsilon_{1}\right) \rightarrow$ $\mathbb{R}^{n},(\mathbf{z}, \varepsilon) \mapsto \mathbf{x}(T, \mathbf{z}, \varepsilon)-\mathbf{z}$. If $(\overline{\mathbf{z}}, \bar{\varepsilon}) \in D \times\left(-\varepsilon_{1}, \varepsilon_{1}\right)$ is such that $f(\overline{\mathbf{z}}, \bar{\varepsilon})=0$, then $\mathbf{x}(t, \overline{\mathbf{z}}, \bar{\varepsilon})$ is a $T$-periodic solution of $\left(3_{\bar{\varepsilon}}\right)$. Clearly the converse is true. Hence the problem of finding $T$-periodic orbits of $\left(3_{\varepsilon}\right)$ close to the periodic orbits with initial conditions in $\mathcal{Z}$ is reduced finding the zeros of $f(\mathbf{z}, \varepsilon)$.

The sets of zeros of $f(\mathbf{z}, \varepsilon)$ and $g(\mathbf{z}, \varepsilon)=M_{\mathbf{z}}^{-1}(T) f(\mathbf{z}, \varepsilon)$ are the same, since $M_{\mathbf{z}}(T)$ is a fundamental matrix. Moreover, following [3], we can compute also

$$
D_{\mathbf{z}} g(\mathbf{z}, \varepsilon)=\left(M_{\mathbf{z}}^{-1}(0)-M_{\mathbf{z}}^{-1}(T)\right)+D_{\mathbf{z}}\left(\int_{0}^{T} M_{\mathbf{z}}^{-1}(t) F_{1}(t, \mathbf{x}(t, \mathbf{z}, 0)) d t\right) \varepsilon+O\left(\varepsilon^{2}\right) .
$$

We note that $g^{-1}(0)=\left(\xi^{\perp} \circ g\right)^{-1}(0) \cap(\xi \circ g)^{-1}(0)$. From $(6)$ we obtain $D_{\mathbf{z}} g\left(\mathbf{z}_{\alpha}, 0\right)=$ $M_{\mathbf{z}_{\alpha}}^{-1}(0)-M_{\mathbf{z}_{\alpha}}^{-1}(T)$ and from $(b)$ such a matrix has in the lower right corner a $(n-m) \times(n-m)$ matrix $\Delta_{\alpha}$ with $\operatorname{det}\left(\Delta_{\alpha}\right) \neq 0$, so by using the Implicit Function 
Theorem, it follows that there are an open neighborhood $U \times\left(-\varepsilon_{2}, \varepsilon_{2}\right)$ of $\mathrm{Cl}(V)$ in $\xi(D) \times\left(-\varepsilon_{1}, \varepsilon_{1}\right)$, an open neighborhood $\mathcal{O}$ of $\beta_{0}(\mathrm{Cl}(V))$ in $\mathbb{R}^{n-m}$ and a unique $\mathcal{C}^{k}$ function $\beta(\alpha, \varepsilon): U \times\left(-\varepsilon_{2}, \varepsilon_{2}\right) \rightarrow \mathcal{O}$ such that $\left(\xi^{\perp} \circ g\right)^{-1}(0) \cap\left(U \times \mathcal{O} \times\left(-\varepsilon_{2}, \varepsilon_{2}\right)\right)$ is exactly the graph of $\beta(\alpha, \varepsilon)$ (for more details see [3]). Now, if we define the function $\delta: U \times\left(-\varepsilon_{2}, \varepsilon_{2}\right) \rightarrow \mathbb{R}$ as $\delta(\alpha, \varepsilon)=(\xi \circ g)(\alpha, \beta(\alpha, \varepsilon), \varepsilon)$, then $\delta$ is a $\mathcal{C}^{k}$ function and $g^{-1}(0) \cap\left(U \times \mathcal{O} \times\left(-\varepsilon_{2}, \varepsilon_{2}\right)\right)=\left\{(\alpha, \beta(\alpha, \varepsilon), \varepsilon) \mid(\alpha, \varepsilon) \in \delta^{-1}(0)\right\}$. Therefore for describing the set $g^{-1}(0)$ in an open neighborhood of $\mathcal{Z}$ in $\mathbb{R}^{n} \times\left(-\varepsilon_{0}, \varepsilon_{0}\right)$, it is sufficient to describe $\delta^{-1}(0)$ in an open neighborhood of $\mathrm{Cl}(V)$ in $\mathbb{R} \times\left(-\varepsilon_{0}, \varepsilon_{0}\right)$.

Since $M_{\mathbf{z}_{\alpha}}^{-1}(0)-M_{\mathbf{z}_{\alpha}}^{-1}(T)$ has in the upper right corner the $m \times(n-m)$ zero matrix and $\delta(\alpha, 0)=0$ in $V \times\left(-\varepsilon_{2}, \varepsilon_{2}\right)$, the function $\delta(\alpha, \varepsilon)$ can be written as $\delta(\alpha, \varepsilon)=\varepsilon \mathcal{F}(\alpha)+\varepsilon^{2} G(\alpha, \varepsilon)$ in $V \times\left(-\varepsilon_{2}, \varepsilon_{2}\right)$, where $\mathcal{F}(\alpha)$ is the function given by (5), see [3]. In addition, if $\widetilde{\delta}(\alpha, \varepsilon)=\mathcal{F}(\alpha)+\varepsilon G(\alpha, \varepsilon)$, then $\delta^{-1}(0)=\widetilde{\delta}^{-1}(0)$.

If there exists $\alpha_{0} \in V$ such that $\widetilde{\delta}\left(\alpha_{0}, 0\right)=\mathcal{F}\left(\alpha_{0}\right)=0$ and $\operatorname{det}\left((\partial \mathcal{F} / \partial \alpha)\left(\alpha_{0}\right)\right) \neq$ 0 then, from the Implicit Function Theorem, there exist $\varepsilon_{3}>0$ small, an open neighborhood $V_{0}$ of $\alpha_{0}$ in $V$ and a unique $\mathcal{C}^{k}$ function $\alpha(\varepsilon):\left(-\varepsilon_{3}, \varepsilon_{3}\right) \rightarrow V_{0}$ such that $\widetilde{\delta}^{-1}(0) \cap\left(V_{0} \times\left(-\varepsilon_{3}, \varepsilon_{3}\right)\right)$ is the graph of $\alpha(\varepsilon)$, which also represent the set $\delta^{-1}(0) \cap\left(V_{0} \times\left(-\varepsilon_{3}, \varepsilon_{3}\right)\right)$. This proves the statement $(i)$.

Moreover if $m=1$ the function $\mathcal{F}(\alpha)$ is of one variable, so we can consider higher order derivatives of it. Suppose that

$$
\frac{\partial \widetilde{\delta}}{\partial \alpha}\left(\alpha_{0}, 0\right)=\mathcal{F}^{\prime}\left(\alpha_{0}\right)=0, \ldots, \frac{\partial^{s-1} \widetilde{\delta}}{\partial \alpha^{s-1}}\left(\alpha_{0}, 0\right)=\mathcal{F}^{(s-1)}\left(\alpha_{0}\right)=0
$$

and $\frac{\partial^{s} \widetilde{\delta}}{\partial \alpha^{s}}\left(\alpha_{0}, 0\right)=\mathcal{F}^{(s)}\left(\alpha_{0}\right) \neq 0$. We want to prove that there are at most $s T$ periodic solutions of system $\left(3_{\varepsilon}\right)$ bifurcating from $\mathbf{x}\left(t, \mathbf{z}_{\alpha_{0}}\right)$. Suppose the contrary, that is suppose that there are at least $s+1 T$-periodic solutions of system $\left(3_{\varepsilon}\right)$ bifurcating from $\mathbf{x}\left(t, \mathbf{z}_{\alpha_{0}}\right)$, then for any integer $j$ there exist $\varepsilon_{j}>0$ and $\eta_{j}>0$, $\varepsilon_{j} \rightarrow 0$ and $\eta_{j} \rightarrow 0$ as $j \rightarrow \infty$, such that the function $g\left(\mathbf{z}, \varepsilon_{j}\right)$ has at least $s+1$ zeros in $\left|\mathbf{z}-\mathbf{z}_{\alpha_{0}}\right|<\eta_{j}$. Equivalently the function $\widetilde{\delta}(\alpha, \varepsilon)$ has at least $s+1$ zeros in $\left|\alpha-\alpha_{0}\right|<\eta_{j}$. By using Rolle's Theorem we find a $\alpha_{j}$ such that $\left|\alpha_{j}-\alpha_{0}\right|<\eta_{j}$ and

$$
\mathcal{F}^{(s)}\left(\alpha_{j}\right)+\varepsilon_{j} \frac{\partial^{s} \widetilde{\delta}}{\partial \alpha^{s}}\left(\alpha_{j}, \varepsilon_{j}\right)=0,
$$

which implies $\mathcal{F}^{(s)}\left(\alpha_{0}\right)=0$ by taking limit as $j \rightarrow \infty$, which is a contradiction. Hence statement $(i i)$ is proved.

Note that from Theorem 2 we get the following remark. Let $X_{0}$ be a vector field in $\mathbb{R}^{q}$, with $q \geq 2$, having an invariant manifold $\mathcal{W}$ consisting of periodic orbits of the same period. Consider the vector field $X_{\varepsilon}=X_{0}+\varepsilon X$ an $\varepsilon$-perturbation of $X_{0}$. Suppose that the differential system associated to $X_{\varepsilon}$ can be reduced to a system in $\mathbb{R}^{n}$ of the form $\left(3_{\varepsilon}\right)$ and that the manifold $\mathcal{W}$ corresponds to a $m$-dimensional manifold foliated by periodic orbits of period $T$. If we can apply Theorem 2 to the reduced system, then each $T$-periodic solution given by this theorem corresponds to a limit cycle of the original system, that is, a limit cycle of $X_{\varepsilon}$ for $\varepsilon$ small. 
Therefore, if $\mathcal{F}(\alpha)$ does not vanish identically, then the number of simple zeros of $\mathcal{F}(\alpha)$ is an upper bound for the number of limit cycles of $X_{\varepsilon}$ bifurcating from the periodic orbits of the manifold $\mathcal{W}$ and each simple zero of $\mathcal{F}(\alpha)$ corresponds to a limit cycle of $X_{\varepsilon}$ for $\varepsilon$ small.

In general to know the maximum number of limit cycles that systems $\left(3_{\varepsilon}\right)$ can have is a very difficult problem. The result described in the previous paragraph gives a partial answer in that direction. That is, the upper bound provided by the simple zeros of the function $\mathcal{F}(\alpha)$, when it is reached, is a lower bound for the maximum number of limit cycles of the perturbed system $\left(3_{\varepsilon}\right)$.

In this paper we will show an explicit implementation of Theorem 2 for computing limit cycles of $\left(1_{\varepsilon}\right)$ which bifurcate from the torus $\mathbb{T}$ of $\left(1_{0}\right)$.

\section{Proof of the results}

The proof of Theorem 1 is based in the following three technical lemmas which are proved later on.

Lemma 4 By using a suitable change of coordinates $(\theta, \phi, r)$, in an open neighborhood of the torus $\mathbb{T}$, system $\left(1_{\varepsilon}\right)$ can be transformed into the form $\left(3_{\varepsilon}\right)$. The transformed system satisfies the conditions $(a)$ and (b) of Theorem 2; and (5) takes the form (2).

Lemma 5 If $P(x, y, z)$ and $Q(x, y, z)$ are polynomials of degree at most $d$, then the expression (2) takes the form

$$
\mathcal{F}(\phi)=a_{0}+\sum_{l=1}^{d+1}\left(a_{l} \cos (l \phi)+b_{l} \sin (l \phi)\right),
$$

where $a_{0}, a_{1}, \ldots, a_{d+1}, b_{1}, \ldots, b_{d+1}$ are functions of the coefficients of $P(x, y, z)$ and $Q(x, y, z)$. If this function does not vanish identically, then it has at most $2(d+1)$ isolated zeros counting multiplicities in $\mathbb{S}^{1}$.

Lemma 6 The following statements hold.

(i) There are polynomials $P(x, y, z)$ and $Q(x, y, z)$ such that the coefficients of the function $\mathcal{F}(\phi)$ given by $(7)$ are all independent.

(ii) For any natural $d$ and any $\nu$ in the set $\{2,4, \ldots, 2(d+1)\}$, we can find polynomials $P(x, y, z)$ and $Q(x, y, z)$ of degree $d$ such that the function $\mathcal{F}(\phi)$ given by $(7)$ has exactly $\nu$ zeros and each one of them is simple.

Proof of Theorem 1. From Lemma 4, system $\left(1_{\varepsilon}\right)$ is reduced to the form $\left(3_{\varepsilon}\right)$ and we can apply Theorem 2 for studying isolated periodic orbits of the reduced system or equivalently limit cycles of the original system $\left(1_{\varepsilon}\right)$. If the maximum of the degrees of $P(x, y, z)$ and $Q(x, y, z)$ is $d$ and the function $\mathcal{F}(\phi)$ does not vanish 
identically, then from Lemma 5 the function $\mathcal{F}(\phi)$ has at most $2(d+1)$ isolated zeros in $\mathbb{S}^{1}$. Hence statement (ii) of Theorem 2 proves statement $(i)$ of Theorem 1.

For proving statement (ii) we need to show that, for any natural $d$ and any $\nu$ in the set $\{2,4, \ldots, 2(d+1)\}$, there are polynomials $P(x, y, z)$ and $Q(x, y, z)$ of degree $d$ such that the function $\mathcal{F}(\phi)$ has exactly $\nu$ zeros in $\mathbb{S}^{1}$ and each one of them is simple. This does not follow directly from Lemma 5 because in general the coefficients of $\mathcal{F}(\phi)$ given by (7) are no independent; however Lemma 6 ensures this property, and applying Theorem 2.(i) and Corollary 3 the statement follows.

Proof of Lemma 4. If $\theta, \phi \in \mathbb{S}^{1}$ and $r \in(0,2)$, then

$$
x=(2+r \cos \theta) \cos \phi, \quad y=(2+r \cos \theta) \sin \phi \quad \text { and } \quad z=r \sin \theta,
$$

is a change of coordinates in $\mathbb{S}^{1} \times \Omega$, with $\Omega:=\left\{(\phi, r) \mid \phi \in \mathbb{S}^{1}, 0<r<2\right\}$. The torus $\mathbb{T}$ corresponds to the points with $r=1$. By using this change of coordinates system $\left(1_{\varepsilon}\right)$ becomes

$$
\begin{aligned}
& \dot{\theta}=1+\varepsilon(\widetilde{R}(\theta, \phi, r) \cos \theta-\sin \theta(\widetilde{P}(\theta, \phi, r) \cos \phi+\widetilde{Q}(\theta, \phi, r) \sin \phi)) / r, \\
& \dot{\phi}=\varepsilon(\widetilde{Q}(\theta, \phi, r) \cos \phi-\widetilde{P}(\theta, \phi, r) \sin \phi) /(2+r \cos \theta), \\
& \dot{r}=r\left(1-r^{2}\right)+\varepsilon \cos \theta(\widetilde{P}(\theta, \phi, r) \cos \phi+\widetilde{Q}(\theta, \phi, r) \sin \phi+\widetilde{R}(\theta, \phi, r)),
\end{aligned}
$$

where $\tilde{Y}(\theta, \phi, r):=Y((2+r \cos \theta) \cos \phi,(2+r \cos \theta) \sin \phi, r \sin \theta)$ for $Y \in\{P, Q, R\}$.

We change the independent variable $t$ of system $\left(8_{\varepsilon}\right)$ by the variable $\theta$, and we obtain the equivalent 2-dimensional system

$$
\begin{aligned}
\phi^{\prime} & =\varepsilon(\widetilde{Q}(\theta, \phi, r) \cos \phi-\widetilde{P}(\theta, \phi, r) \sin \phi) /(2+r \cos \theta)+O\left(\varepsilon^{2}\right), \\
r^{\prime} & =r\left(1-r^{2}\right)+\varepsilon S(\theta, \phi, r)+O\left(\varepsilon^{2}\right),
\end{aligned}
$$

where

$$
\begin{aligned}
S(\theta, \phi, r):= & \left(\cos \theta-\left(1-r^{2}\right) \sin \theta\right)(\widetilde{P}(\theta, \phi, r) \cos \phi+\widetilde{Q}(\theta, \phi, r) \sin \phi) \\
& +\left(2-r^{2}\right) \widetilde{R}(\theta, \phi, r) \cos \theta,
\end{aligned}
$$

which is defined in $\Omega$. The prime denotes the differentiation with respect to the variable $\theta$.

If we use the notation $\mathbf{x}:=\left(\begin{array}{c}\phi \\ r\end{array}\right)$ then $\left(9_{\varepsilon}\right)$ can be written as

$$
\mathbf{x}^{\prime}(\theta)=F_{0}(\mathbf{x})+\varepsilon F_{1}(\theta, \mathbf{x})+\varepsilon^{2} F_{2}(\theta, \mathbf{x}, \varepsilon) .
$$

It is clear that $F_{0}, F_{1}: \mathbb{R} \times \Omega \rightarrow \Omega$ and $F_{2}: \mathbb{R} \times \Omega \times\left(-\varepsilon_{0}, \varepsilon_{0}\right) \rightarrow \Omega$ are $2 \pi$-periodic in $\theta$ and analytic. Thus $\left(9_{\varepsilon}\right)$ has the form $\left(3_{\varepsilon}\right)$.

Consider the subset

$$
\mathcal{Z}:=\left\{\mathbf{z}_{\phi}=(\phi, 1) \mid \phi \in \mathbb{S}^{1}\right\}
$$


of $\Omega$. The solution of $\left(9_{\varepsilon}\right)$ passing through the point $\mathbf{z}_{\phi}$ is $\mathbf{x}\left(\theta, \mathbf{z}_{\phi}\right)=\left(\begin{array}{c}\phi \\ 1\end{array}\right)$ which is constant, hence $2 \pi$-periodic in $\theta$. Therefore $\mathcal{Z}$ is an invariant 1-dimensional manifold foliated by periodic orbits of the unperturbed system $\left(9_{0}\right)$ (in fact, singular points), which corresponds to the invariant torus $\mathbb{T}$ of system $\left(8_{0}\right)$.

The variational system corresponding to the unperturbed system $\left(9_{0}\right)$ along the solutions of $\mathcal{Z}$ is

$$
\left(\begin{array}{c}
\phi^{\prime} \\
r^{\prime}
\end{array}\right)=\left(\begin{array}{cc}
0 & 0 \\
0 & -2
\end{array}\right)\left(\begin{array}{c}
\phi \\
r
\end{array}\right)
$$

whose solution is $\phi(\theta)=$ constant and $r(\theta)=e^{-2 \theta}$. Hence the fundamental matrix $M_{\mathbf{z}_{\phi}}$ is

$$
M_{\mathbf{z}_{\phi}}(\theta)=\left(\begin{array}{cc}
1 & 0 \\
0 & e^{-2 \theta}
\end{array}\right) .
$$

Then we have

$$
M_{\mathbf{z}_{\phi}}^{-1}(\theta)=\left(\begin{array}{cc}
1 & 0 \\
0 & e^{2 \theta}
\end{array}\right)
$$

whence

$$
M_{\mathbf{z}_{\phi}}^{-1}(0)-M_{\mathbf{z}_{\phi}}^{-1}(2 \pi)=\left(\begin{array}{cc}
0 & 0 \\
0 & 1-e^{4 \pi}
\end{array}\right) .
$$

Therefore taking $V=\mathbb{S}^{1}$ and $\beta_{0}: \mathbb{S}^{1} \rightarrow(0,2)$ the constant function 1 , it is clear that system $\left(9_{\varepsilon}\right)$ satisfies the conditions $(a)$ and $(b)$ of Theorem 2 .

In this case (5) takes the form

$$
\begin{aligned}
& \mathcal{F}(\phi):=\xi\left(\int_{0}^{2 \pi}\left(\begin{array}{cc}
1 & 0 \\
0 & e^{2 \theta}
\end{array}\right) F_{1}\left(\theta, \mathbf{x}\left(\theta, \mathbf{z}_{\phi}\right)\right) d \theta\right) \\
& =\xi\left(\int_{0}^{2 \pi}\left(\begin{array}{cc}
1 & 0 \\
0 & e^{2 \theta}
\end{array}\right)\left(\frac{\widetilde{Q}(\theta, \phi, 1) \cos \phi-\widetilde{P}(\theta, \phi, 1) \sin \phi}{2+\cos \theta}\right) d \theta\right) .
\end{aligned}
$$

That is exactly (2).

For proving Lemma 5 we first recall a property on Chebyshev systems and some technical results.

Definition 7 The set of $j+1$ real functions $f_{0}(x), f_{1}(x), \ldots, f_{j}(x)$ defined in a compact Hausdorff topological set $A$ forms a Chebyshev system in $A$ if any nontrivial linear combination $a_{0} f_{0}(x)+a_{1} f_{1}(x)+\cdots+a_{j} f_{j}(x)$ has at most $j$ zeros in $A$ counting multiplicities.

Proposition 8 Suppose that the set of real functions $f_{0}(x), f_{1}(x), \ldots, f_{j}(x)$ forms a Chebyshev system in $A$. If $x_{0}, x_{1}, \ldots, x_{j}$ are $j+1$ different points in $A$ and $c_{0}, c_{1}, \ldots, c_{j}$ are $j+1$ arbitrary real numbers, then the system of equations

$$
a_{0} f_{0}\left(x_{i}\right)+a_{1} f_{1}\left(x_{i}\right)+\cdots+a_{j} f_{j}\left(x_{i}\right)=c_{i}, \quad i=0,1, \ldots, j,
$$

has a unique solution for $a_{0}, a_{1}, \ldots, a_{j}$. 
For a proof of Proposition 8 see [11, pp. 24].

Lemma 9 If $d$ is a natural then

$$
\sum_{i+j=0}^{d} c_{i j} \cos ^{i} \phi \sin ^{j} \phi=\tilde{a}_{0}+\sum_{l=1}^{d}\left(\tilde{a}_{l} \cos (l \phi)+\tilde{b}_{l} \sin (l \phi)\right),
$$

where $c_{i j} \in \mathbb{R}$ and $\tilde{a}_{0}, \tilde{a}_{1}, \ldots, \tilde{a}_{d}, \tilde{b}_{1}, \ldots, \tilde{b}_{d}$ are functions of $c_{i j}$, with $0 \leq i+j \leq d$.

Proof. Equality (10) is satisfied trivially for $d=0$ and $d=1$. If the equality (10) holds for $k=d$ we should prove the assertion for $k=d+1$.

$$
\sum_{i+j=0}^{d+1} c_{i j} \cos ^{i} \phi \sin ^{j} \phi=\sum_{i+j=0}^{d} c_{i j} \cos ^{i} \phi \sin ^{j} \phi+\sum_{i+j=d} c_{i j} \cos ^{i} \phi \sin ^{j} \phi .
$$

By hypothesis of induction

$$
\sum_{i+j=0}^{d} c_{i j} \cos ^{i} \phi \sin ^{j} \phi=\bar{a}_{0}+\sum_{l=1}^{d}\left(\bar{a}_{l} \cos (l \phi)+\bar{b}_{l} \sin (l \phi)\right),
$$

where $\bar{a}_{0}, \bar{a}_{1}, \ldots, \bar{a}_{d}, \bar{b}_{1}, \ldots, \bar{b}_{d}$ are functions of $c_{i j}$, with $0 \leq i+j \leq d$. On the other hand

$$
\sum_{i+j=d} c_{i j} \cos ^{i} \phi \sin ^{j} \phi=\cos \phi\left(\sum_{i+j=d, i \geq 1} c_{i j} \cos ^{i} \phi \sin ^{j} \phi\right)+c_{0, d+1} \sin ^{d+1} \phi .
$$

By applying again the hypothesis of induction we get

$$
\begin{gathered}
\cos \phi \sum_{i+j=d, i \geq 1} c_{i j} \cos ^{i} \phi \sin ^{j} \phi=\bar{\lambda}_{0} \cos \phi+\sum_{l=1}^{d}\left(\bar{\lambda}_{l} \cos \phi \cos (l \phi)+\bar{\sigma}_{l} \cos \phi \sin (l \phi)\right), \\
c_{0, d+1} \sin ^{d+1} \phi=c_{0, d+1} \sin \phi \sin ^{d} \phi=\bar{\mu}_{0} \sin \phi+\sum_{l=1}^{d}\left(\bar{\mu}_{l} \sin \phi \cos (l \phi)+\bar{\rho}_{l} \sin \phi \sin (l \phi)\right),
\end{gathered}
$$

where $\bar{\lambda}_{0}, \bar{\lambda}_{1}, \ldots, \bar{\lambda}_{d}, \bar{\sigma}_{1}, \ldots, \bar{\sigma}_{d}$ are functions of $c_{i j}$, with $i+j=d, i \geq 1$ and $\bar{\mu}_{0}, \bar{\mu}_{1}, \ldots, \bar{\mu}_{d}, \bar{\rho}_{1}, \ldots, \bar{\rho}_{d}$ are functions of $c_{0, d+1}$. By applying the formulas

$$
\begin{aligned}
\cos \phi \cos (l \phi) & =(\cos ((l+1) \phi)+\cos ((l-1) \phi)) / 2, \\
\cos \phi \sin (l \phi) & =(\sin ((l+1) \phi)-\sin ((l-1) \phi)) / 2, \\
\sin \phi \cos (l \phi) & =(\sin ((l+1) \phi)+\sin ((l-1) \phi)) / 2, \\
\sin \phi \sin (l \phi) & =(\cos ((l-1) \phi)-\cos ((l+1) \phi)) / 2,
\end{aligned}
$$


we obtain

$$
\begin{gathered}
\cos \phi \sum_{i+j=d, i \geq 1} c_{i j} \cos ^{i} \phi \sin ^{j} \phi=\hat{\lambda}_{0}+\sum_{l=1}^{d+1}\left(\hat{\lambda}_{l} \cos (l \phi)+\hat{\sigma}_{l} \sin (l \phi)\right), \\
c_{0, d+1} \sin ^{d+1} \phi=c_{0, d+1} \sin \phi \sin ^{d} \phi=\hat{\mu}_{0}+\sum_{l=1}^{d+1}\left(\hat{\mu}_{l} \cos (l \phi)+\hat{\rho}_{l} \sin (l \phi)\right),
\end{gathered}
$$

where $\hat{\lambda}_{0}, \hat{\lambda}_{1}, \ldots, \hat{\lambda}_{d+1}, \hat{\sigma}_{1}, \ldots, \hat{\sigma}_{d+1}$ are functions of $c_{i j}$, with $i+j=d, i \geq 1$ and $\hat{\mu}_{0}, \hat{\mu}_{1}, \ldots, \hat{\mu}_{d+1}, \hat{\rho}_{1}, \ldots, \hat{\rho}_{d+1}$ are functions of $c_{0, d+1}$.

Hence (13) can be written as

$$
\sum_{i+j=d} c_{i j} \cos ^{i} \phi \sin ^{j} \phi=\hat{a}_{0}+\sum_{l=1}^{d+1}\left(\hat{a}_{l} \cos (l \phi)+\hat{b}_{l} \sin (l \phi)\right),
$$

where $\hat{a}_{0}, \hat{a}_{1}, \ldots, \hat{a}_{d+1}, \hat{b}_{1}, \ldots, \hat{b}_{d+1}$ are functions of $c_{i j}$, with with $i+j=d$. By using (15) and (12) we have that (11) takes the form

$$
\sum_{i+j=0}^{d+1} c_{i j} \cos ^{i} \phi \sin ^{j} \phi=\tilde{a}_{0}+\sum_{l=1}^{d+1}\left(\tilde{a}_{l} \cos (l \phi)+\tilde{b}_{l} \sin (l \phi)\right),
$$

where $\tilde{a}_{0}, \tilde{a}_{1}, \ldots, \tilde{a}_{d+1}, \tilde{b}_{1}, \ldots, \tilde{b}_{d+1}$ are functions of $c_{i j}$, with $0 \leq i+j \leq d$.

Proof of Lemma 5. Suppose that

$$
P(x, y, z)=\sum_{i+j+k=0}^{d} p_{i j k} x^{i} y^{j} z^{k}, \quad Q(x, y, z)=\sum_{i+j+k=0}^{d} q_{i j k} x^{i} y^{j} z^{k},
$$

then

$$
\begin{aligned}
& \frac{\widetilde{P}(\theta, \phi, 1)}{2+\cos \theta}=\sum_{i+j=0}^{d}\left(\sum_{k=0}^{d-i-j} p_{i j k}(2+\cos \theta)^{i+j-1} \sin ^{k} \theta\right) \cos ^{i} \phi \sin ^{j} \phi, \\
& \frac{\widetilde{Q}(\theta, \phi, 1)}{2+\cos \theta}=\sum_{i+j=0}^{d}\left(\sum_{k=0}^{d-i-j} q_{i j k}(2+\cos \theta)^{i+j-1} \sin ^{k} \theta\right) \cos ^{i} \phi \sin ^{j} \phi .
\end{aligned}
$$

Therefore

$$
\begin{aligned}
& \int_{0}^{2 \pi} \frac{\widetilde{P}(\theta, \phi, 1)}{2+\cos \theta} d \theta=\sum_{i+j=0}^{d} \tilde{p}_{i j} \cos ^{i} \phi \sin ^{j} \phi \\
& \int_{0}^{2 \pi} \frac{\widetilde{Q}(\theta, \phi, 1)}{2+\cos \theta} d \theta=\sum_{i+j=0}^{d} \tilde{q}_{i j} \cos ^{i} \phi \sin ^{j} \phi
\end{aligned}
$$


where

$$
\begin{aligned}
& \tilde{p}_{i j}:=\sum_{k=0}^{d-i-j} p_{i j k} \int_{0}^{2 \pi}(2+\cos \theta)^{i+j-1} \sin ^{k} \theta d \theta, \\
& \tilde{q}_{i j}:=\sum_{k=0}^{d-i-j} q_{i j k} \int_{0}^{2 \pi}(2+\cos \theta)^{i+j-1} \sin ^{k} \theta d \theta .
\end{aligned}
$$

From Lemma 9 we have

$$
\sum_{i+j=0}^{d} \tilde{p}_{i j} \cos ^{i} \phi \sin ^{j} \phi=\tilde{a}_{0}+\sum_{l=1}^{d}\left(\tilde{a}_{l} \cos (l \phi)+\tilde{b}_{l} \sin (l \phi)\right)
$$

and

$$
\sum_{i+j=0}^{d} \tilde{q}_{i j} \cos ^{i} \phi \sin ^{j} \phi=\hat{a}_{0}+\sum_{l=1}^{d}\left(\hat{a}_{l} \cos (l \phi)+\hat{b}_{l} \sin (l \phi)\right) .
$$

Where $\tilde{a}_{0}, \tilde{a}_{1}, \ldots, \tilde{a}_{d}, \tilde{b}_{1}, \ldots, \tilde{b}_{d}$ are functions of $\tilde{p}_{i j}$, with $0 \leq i+j \leq d$, hence using (18) they are functions of the coefficients of $P(x, y, z)$. Analogously the coefficients $\hat{a}_{0}, \hat{a}_{1} \ldots, \hat{a}_{d}, \hat{b}_{1}, \ldots, \hat{b}_{d}$ are functions of $\tilde{q}_{i j}$, with $0 \leq i+j \leq d$, hence using (19) they are functions of the coefficients of $Q(x, y, z)$.

In short, from (16), (17), (20) and (21) we obtain that the function $\mathcal{F}(\phi)$ given by (2) takes the form

$$
\begin{aligned}
\mathcal{F}(\phi)= & \cos \phi\left(\hat{a}_{0}+\sum_{l=1}^{d}\left(\hat{a}_{l} \cos (l \phi)+\hat{b}_{l} \sin (l \phi)\right)\right)- \\
& \sin \phi\left(\tilde{a}_{0}+\sum_{l=1}^{d}\left(\tilde{a}_{l} \cos (l \phi)+\tilde{b}_{l} \sin (l \phi)\right)\right) .
\end{aligned}
$$

If we apply the formulas given by (14) then (22) is reduced to (7).

Since the functions $1, \cos \phi, \sin \phi, \ldots, \cos ((d+1) \phi), \sin ((d+1) \phi)$ form a Chebyshev system in $\mathbb{S}^{1}$ (see for instance [11, pp. 23-24]), the function $\mathcal{F}(\phi)$ has at most $2(d+1)$ zeros in $\mathbb{S}^{1}$ if it does not vanish identically.

Proof of Lemma 6. We will construct polynomials $P(x, y, z)$ and $Q(x, y, z)$ of degree $d$ such that the coefficients of $\mathcal{F}(\phi)$ given by (7) are all independent.

Taking $G_{0}(x, y)=1$ and $H_{0}(x, y)=0$ we define recursively

$$
\begin{aligned}
& G_{d+1}(x, y)=x G_{d}(x, y)-y H_{d}(x, y), \\
& H_{d+1}(x, y)=y G_{d}(x, y)+x H_{d}(x, y) .
\end{aligned}
$$

Note that $G_{d}(x, y)$ and $H_{d}(x, y)$ are polynomials of degree $d$. If we define

$$
\begin{aligned}
& \widetilde{G}_{d}(\theta, \phi, r):=G_{d}((2+r \cos \theta) \cos \phi,(2+r \cos \theta) \sin \phi), \\
& \widetilde{H}_{d}(\theta, \phi, r):=H_{d}((2+r \cos \theta) \cos \phi,(2+r \cos \theta) \sin \phi),
\end{aligned}
$$


then it is easy to see that

$$
\begin{aligned}
\widetilde{G}_{d}(\theta, \phi, r) & =(2+r \cos \theta)^{d} \cos (d \phi), \\
\widetilde{H}_{d}(\theta, \phi, r) & =(2+r \cos \theta)^{d} \sin (d \phi) .
\end{aligned}
$$

Now we define the following polynomials in $\mathbb{R}^{3}$ which only depend on the variables $x$ and $y$

$$
\begin{aligned}
& P_{d}(x, y, z)=-a_{0} y+\sum_{l=0}^{d} \frac{-b_{l+1} G_{l}+a_{l+1} H_{l}}{c_{l+1}}, \\
& Q_{d}(x, y, z)=a_{0} x+\sum_{l=0}^{d} \frac{a_{l+1} G_{l}+b_{l+1} H_{l}}{c_{l+1}}
\end{aligned}
$$

where $a_{0}, \ldots, a_{d+1}, b_{1}, \ldots, b_{d+1} \in \mathbb{R}$ and

$$
c_{l+1}:=\int_{0}^{2 \pi}(2+\cos \theta)^{l-1} d \theta \neq 0, \quad l=0,1, \ldots, d .
$$

We note that the polynomials $P_{0}(x, y, z), Q_{0}(x, y, z), P_{1}(x, y, z)$ and $Q_{1}(x, y, z)$ are of degree 1 . For $d \geq 1$ the polynomials $P_{d}(x, y, z)$ and $Q_{d}(x, y, z)$ are of degree $d$.

Then

$$
\begin{aligned}
\widetilde{P}_{d}(\theta, \phi, 1) \sin \phi= & -a_{0}(2+\cos \theta) \sin ^{2} \phi \\
& +\sum_{l=0}^{d} \frac{\sin \phi\left(-b_{l+1} \widetilde{G}_{l}(\theta, \phi, 1)+a_{l+1} \widetilde{H}_{l}(\theta, \phi, 1)\right)}{c_{l+1}}, \\
\widetilde{Q}_{d}(\theta, \phi, 1) \cos \phi= & a_{0}(2+\cos \theta) \cos ^{2} \phi \\
& +\sum_{l=0}^{n} \frac{\cos \phi\left(a_{l+1} \widetilde{G}_{l}(\theta, \phi, 1)+b_{l+1} \widetilde{H}_{l}(\theta, \phi, 1)\right)}{c_{l+1}}
\end{aligned}
$$

If we replace $\widetilde{P}_{d}(\theta, \phi, 1) \sin \phi$ and $\widetilde{Q}_{d}(\theta, \phi, 1) \cos \phi$ in (2) we obtain

$$
\begin{aligned}
\mathcal{F}(\phi)= & \int_{0}^{2 \pi}\left[a_{0}+\frac{a_{1}}{c_{1}(2+\cos \theta)} \cos \phi+\frac{b_{1}}{c_{1}(2+\cos \theta)} \sin \phi\right. \\
& +\sum_{l=1}^{d}\left[a_{l+1}(\cos \phi \cos (l \phi)-\sin \phi \sin (l \phi))\right. \\
& \left.\left.+b_{l+1}(\cos \phi \sin (l \phi)+\sin \phi \cos (l \phi))\right] \frac{(2+\cos \theta)^{l-1}}{c_{l+1}}\right] d \theta .
\end{aligned}
$$

Using

$$
\begin{aligned}
& \cos ((l+1) \phi)=\cos \phi \cos (l \phi)-\sin \phi \sin (l \phi), \\
& \sin ((l+1) \phi)=\cos \phi \sin (l \phi)+\sin \phi \cos (l \phi),
\end{aligned}
$$


and (24) we get

$$
\mathcal{F}(\phi)=a_{0}+\sum_{l=1}^{d+1}\left(a_{l} \cos (l \phi)+b_{l} \sin (l \phi)\right) .
$$

Since the coefficients $a_{0}, a_{1}, \ldots, a_{d+1}, b_{1}, \ldots, b_{d+1}$ were chosen independent, the statement $(i)$ is proved.

For the proof of $(i i)$ we consider two cases.

Case $d \geq 1$. Let $\nu$ be in the set $\{2,4, \ldots, 2(d+1)\}$. By using the polynomials $P_{\nu / 2-1}(x, y, z)$ and $Q_{\nu / 2-1}(x, y, z)$ of degree $d=\nu / 2-1$ given by $(23),(25)$ becomes

$$
\mathcal{F}(\phi)=a_{0}+\sum_{l=1}^{\nu / 2}\left(a_{l} \cos (l \phi)+b_{l} \sin (l \phi)\right) .
$$

Since the $\nu+1$ functions $1, \cos \phi, \sin \phi, \ldots, \cos ((\nu / 2) \phi), \sin ((\nu / 2) \phi)$ form a Chebyshev system in $\mathbb{S}^{1}$, from Proposition 8 we know that fixed $\nu$ different points $\phi_{1}, \ldots, \phi_{\nu}$ in $\mathbb{S}^{1}$ we can find a unique solution for $a_{0}, a_{1}, \ldots, a_{\nu / 2}, b_{1}, \ldots, b_{\nu / 2}$ such that the function $\mathcal{F}(\phi)$ given by $(26)$ has a simple zero at each one of the points $\phi_{1}, \ldots, \phi_{\nu}$. Let $\bar{a}_{0}, \bar{a}_{1}, \ldots, \bar{a}_{\nu / 2}, \bar{b}_{1}, \ldots, \bar{b}_{\nu / 2}$ be such solution.

Now we consider the polynomials $P_{d}(x, y, z)$ and $Q_{d}(x, y, z)$ given by $(23)$ such that

$$
a_{0}=\bar{a}_{0}, a_{1}=\bar{a}_{1}, \ldots, a_{\nu / 2}=\bar{a}_{\nu / 2}, b_{1}=\bar{b}_{1}, \ldots, b_{\nu / 2}=\bar{b}_{\nu / 2} .
$$

If we choose the rest of the coefficients of $P_{d}(x, y, z)$ and $Q_{d}(x, y, z)$ sufficiently small then the function $\mathcal{F}(\phi)$ given by (25) has exactly $\nu$ zeros and each one of them is simple.

Case $d=0$. Then $\nu \in\{2,4, \ldots, 2(d+1)\}$ only can takes the value $\nu=2$. By considering $d=0$ and $a_{0}=0$ in (23), we obtain the polynomials $P_{0}(x, y, z)=b_{1}$ and $Q_{0}(x, y, z)=a_{1}$ of degree $d=0$ and $\mathcal{F}(\phi)=a_{1} \cos \phi+b_{1} \sin \phi$. By choosing, for instance, $a_{1}=1$ and $b_{1}=0, \mathcal{F}(\phi)=\cos \phi$ has exactly two zeros and each one of them is simple.

These two cases prove statement $(i i)$.

Remark 10 From the structure of $\mathcal{F}(\phi)$ it cannot have an odd number of zeros and each one of them simple. Therefore, for $\nu$ odd is not easy to find polynomials $P(x, y, z)$ and $Q(x, y, z)$ such that system $\left(1_{\varepsilon}\right)$ has exactly $\nu$ limit cycles bifurcating from the periodic orbits of the torus $\mathbb{T}$ of $\left(1_{0}\right)$.

\section{References}

[1] V. I. Arnold, Mathematical Methods of Classical Mechanics, second edition, Graduate Texts in Mathematics 60, Springer-Verlag, New York. 1989.

[2] V. I. Arnold and A. Avez, Problèmes Ergodiques de la Mécanique Classique, Monographies Internationales de Mathématiques Modernes, No. 9 GauthierVillars, Éditeur, Paris, 1967. 
[3] A. Buică, J. P. Françoise and J. Llibre, Periodic solutions of nonlinear periodic differential systems with a small parameter, Comm. on Pure and Appl. Anal. 6 (2007), 103-111.

[4] C. Buzzi, J. Llibre, J. Medrado and J. Torregrosa, Bifurcation of limit cycles from a center in $\mathbb{R}^{4}$ in ressonance 1:N, Dynamical Systems: An International Journal, 24 (2009), 123-137.

[5] C. Christopher and C. Li, Limit Cycles of Differential Equations, Advanced Courses in Mathematics, CRM Barcelona, Birkhäuser Verlag, Basel. 2007.

[6] A. Cima, J. Llibre and M. Teixeira, Hopf bifurcation in presence of 1:3 resonance, Adv. Nonlinear Stud. 8 (2008), 619-632.

[7] P. Leszczynski and H. Zoladek, Limit cycles appearing after perturbation of certain multidimensional vector fields, J. Dynam. Differential Equations 13 (2001), 689-709.

[8] J. Llibre and M. A. Teixeira, Limit cycles bifurcating from a 2-dimensional isochronous cylinder, Appl. Math. Lett. 22 (2009), 1231-1234.

[9] J. Llibre and M. A. Teixeira, Limit Cycles for a Mechanical System Coming from the Perturbation of a Four-Dimensional Linear Center, J. Dynam. Differential Equations 18 (2006), 931-941.

[10] J. Llibre. M. A. Teixeira and J. Torregrosa, Limit cycles bifurcating from a $k$-dimensional isochronous center contained in $\mathbb{R}^{n}$ with $k \leq n$, Mathematical Physics, Analysis and Geometry 10 (2007), 237-249.

[11] G. G. Lorentz, Approximation of Functions, second edition, Chelsea Publishing Co., New York, 1986.

[12] I. G. Malkin, Some problems of the theory of nonlinear oscillations, (Russian) Gosudarstv. Izdat. Tehn.-Teor. Lit., Moscow, 1956.

[13] M. Roseau, Vibrations non linéaires et théorie de la stabilité, (French) Springer Tracts in Natural Philosophy, Vol.8, Springer-Verlag, Berlin-New York. 1966.

[14] J. A. Sanders and F. Verhulst, Averaging Methods in Nonlinear Dynamical Systems, Applied Mathematical Sciences 59. Springer-Verlag, New York. 1985.

[15] F. Verhulst, Nonlinear Differential Equations and Dynamical Systems, second edition, Universitext, Springer-Verlag, Berlin. 1996. 